\title{
Probing Nanotube-Nanopore Interactions
}

\section{Citation}

King, G. M., and J. A. Golovchenko. 2005. Probing Nanotube-Nanopore Interactions. Physical Review Letters 95, no. 21: 21603-1-216103-4. doi:10.1103/physrevlett.95.216103.

\section{Published Version}

doi:10.1103/PhysRevLett.95.216103

\section{Permanent link}

http://nrs.harvard.edu/urn-3:HUL.InstRepos:29405818

\section{Terms of Use}

This article was downloaded from Harvard University's DASH repository, and is made available under the terms and conditions applicable to Other Posted Material, as set forth at http:// nrs.harvard.edu/urn-3:HUL.InstRepos:dash.current.terms-of-use\#LAA

\section{Share Your Story}

The Harvard community has made this article openly available.

Please share how this access benefits you. Submit a story.

\section{Accessibility}




\title{
Probing Nanotube-Nanopore Interactions
}

\author{
G. M. King ${ }^{1}$ and J. A. Golovchenko ${ }^{1,2}$ \\ ${ }^{1}$ Department of Physics, Harvard University, Cambridge, Massachusetts 02138, USA \\ ${ }^{2}$ Division of Engineering and Applied Sciences, Harvard University, Cambridge, Massachusetts 02138, USA
}

(Received 2 May 2005; published 18 November 2005)

\begin{abstract}
We demonstrate a new nanoscale system consisting of a nanotube threaded through a nanopore in aqueous solution. Its electrical and mechanical properties are sensitive to experimentally controllable conformational changes on sub-Angstrom length scales. Ionic current transport through a nanopore is significantly suppressed by the threading nanotube and the mechanical interactions between the nanotube and pore are accounted for by a folding geometry. The experiments provide first measurements of the longitudinal resolution and metrology of a solid-state nanopore "microscope." This new nanostructure provides a means to study molecule-nanotube interactions in conducting ionic solutions as well as geometrical and surface properties of nanopores and nanotubes.
\end{abstract}

PACS numbers: 81.07. $-\mathrm{b}$

Nanoscale science has advanced through the development of experimental tools, such as the scanning tunneling microscope (STM) [1], atomic force microscope (AFM) [2], and scanning ion conductance microscope [3] that probe and manipulate matter on atomic and molecular scales [4]. The discovery of new nanoscale material structures, like carbon "Bucky balls" and nanotubes, has also stimulated this field [5]. A nanoscale structure of increasing interest is a nanopore traversing a thin insulating solidstate membrane. The development and study of these structures has been inspired by advances in understanding the function of nanopores in biological membranes of living cells [6] and by their use in elegant single molecule experiments $[7,8]$. Robust solid-state nanopores can now be fabricated with tunable diameters [9] and their utility has been demonstrated for sensing single molecules and studying their conformations with subnanometer transverse resolution in a "nanopore microscope" [10]. To date, such single molecule studies have been limited by excessive electrophoretically driven molecular speeds in the nanopores and dominated by stochastic aspects of molecule delivery and translocation.

Here we report the first study of deterministically controlled interactions between a nanopore and a translocating rigid solid-state "molecule" in the form of a carbon nanotube. We demonstrate that an electrically monitored solidstate nanopore can be controllably threaded with a carbon nanotube to achieve electronic sensitivity to picometer length scale mechanical displacements in a fluid. The sensitivity of this mechano-electrical transduction within our solid-state nanoscale system exceeds that in the highly refined biological mechanism of hearing by more than a factor of ten at similar bandwidths [11]. The sensitivity and rigidity of this nanostructure along with its controllable molecular translocation speed evokes several new approaches to single molecule sensing with nanopores.

Figure 1 shows a schematic of the experiment. It consists of a single nanopore in a freestanding membrane of silicon nitride. The membrane separates two reservoirs of conducting electrolyte solution [1 $\mathrm{M} \mathrm{KCl}, 10 \mathrm{mM}$ TRIS- $\mathrm{HCl}$, $1 \mathrm{mM}$ EDTA, $p \mathrm{H}$ 8]. Electrical contact to the solution is achieved though $\mathrm{Ag} / \mathrm{AgCl}$ electrodes shown in black (Fig. 1). A carbon nanotube to be threaded through the nanopore is affixed to the tip of an AFM cantilever. Both are attached to the piezoelectric translation generating elements and optical deflection sensing functionality of an AFM. The motion of the cantilever perpendicular to the membrane surface is controlled by the " $z$ piezo" of the AFM. Lateral drift in the $x-y$ position of the sample and its fluidic chamber is reduced by a closed loop translation table (Npoint Inc., Madison, Wisconsin).

Nanopores were fabricated by the ion sculpting method [9]. A transmission electron microscope (TEM) image of the $13 \mathrm{~nm}$ pore used in this work is shown in Fig. 2(a). Carbon nanotubes were grown directly [12] onto Fe catalyzed Si AFM tips [13] using a vapor deposition method [14]. To enhance their mechanical stability, the nanotubes are affixed to the AFM tip with carbonaceous films deposited in a scanning electron microscope $(3 \mathrm{keV}$, dose $\sim 1 \mathrm{C} / \mathrm{cm}^{2}$ ) [15]. Figure 2(b) shows a TEM image of the $105 \mathrm{~nm}$ long, $5 \mathrm{~nm}$ diameter nanotube mounted on the AFM tip which was used in this work.

The AFM scanning feature is used to align the nanotube with the nanopore. Figure 2 (c) shows a $375 \times 187 \mathrm{~nm}$ contact mode topographic scan of the nanopore under

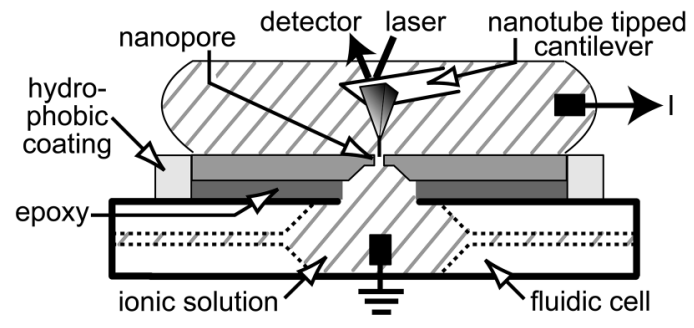

FIG. 1. Schematic of the apparatus. 

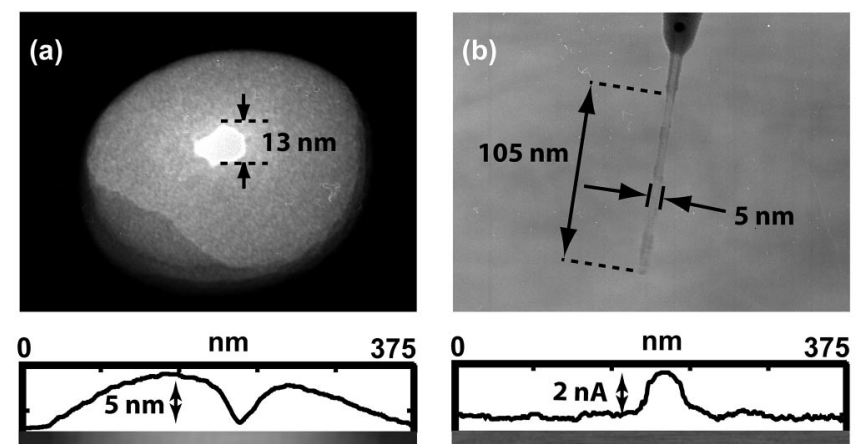

(c) topography
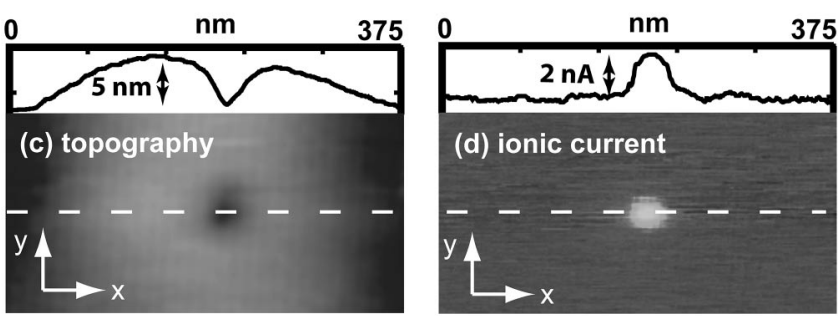

(d) ionic current

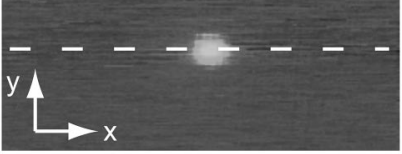

FIG. 2. (a) and (b) TEM images of the nanopore and the nanotube tip. (c) In-fluid topographic AFM image of the nanopore. (d) Ionic current image.

electrolyte solution with the nanotube-tipped AFM. Here the imaging force is set to $50 \mathrm{nN}$, and the fast raster axis is $+x$ with a tip velocity of $800 \mathrm{~nm} / \mathrm{s}$. The nanopore is the topographically low, dark circular region in the center of the image. Data from a line scan (dashed line) through the nanopore region are displayed above the image [16]. A simultaneously taken "image" of the nanopore ionic current blockage magnitude (bias voltage $V=-200 \mathrm{mV}$, open pore current $I_{o}=-7.9 \mathrm{nA}, 10 \mathrm{KHz}$ bandwidth) is displayed in Fig. 2(d). As the nanotube-tipped AFM probe passes over the nanopore, the measured ionic current $I$ is reduced. A line scan of the current modulation $\left|\Delta I_{b}\right|$ (where $\Delta I_{b}=I_{o}-I$ ) is shown above the ionic image.

Nanotube translocation through the nanopore is achieved with the AFM tip centered over the nanopore and the $x$ and $y$ positions held fixed. The $z$ piezo affixed to the base of the AFM cantilever is then withdrawn $\sim 100 \mathrm{~nm}$ from the surface [17] to allow the nanotube tip to straighten and to align itself with the funnel-like entrance to the nanopore. The $z$ piezo is then used to drive the nanotube into and out of the nanopore repeatedly while cantilever deflection and ionic current through the pore are monitored. Lateral instrumental drift currently limits the number of nanotube threading scans that can be made in a single run [18]. Successful nanotube translocation often requires multiple attempts before a sufficiently accurate alignment is obtained.

Figure 3 shows data obtained during two consecutive nanotube threadings versus elapsed time. Curve A shows the current blockage $\Delta I_{b}$. Curve $\mathrm{B}$ shows the advancing and receding AFM $z$ piezo drive displacement $Z_{\text {piezo }}$, and curve $\mathrm{C}$ shows the cantilever tip displacement $Z_{\text {tip }} . Z_{\text {tip }}$ is defined as the difference between $Z_{\text {piezo }}$ and the relative displacement of the cantilever tip determined from the AFM optical deflection sensor. It is not possible to monitor

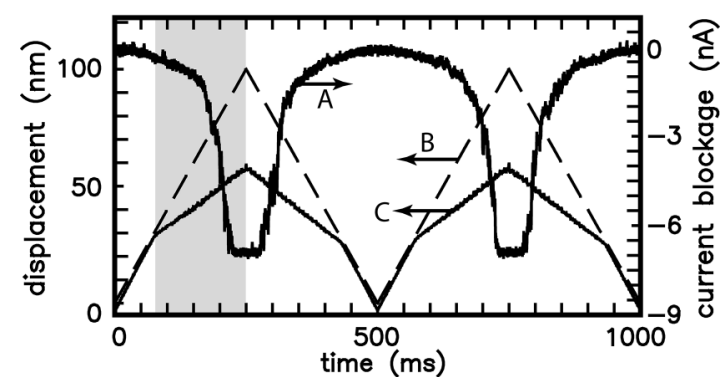

FIG. 3. Ionic current blockage (A) and nanotube tip displacement $(\mathrm{C})$ plotted simultaneously versus time. The dashed line (B) indicates $Z_{\text {piezo }}$.

the displacement of the nanotube in the nanopore directly. $Z_{\text {tip }}$ is the closest observable to this quantity. Also the force the cantilever experiences and transmits to the nanotube is determined by the cantilever spring constant $(k=$ $2 \mathrm{nN} / \mathrm{nm})$ and the difference between $Z_{\text {tip }}$ and $Z_{\text {piezo }}$. In the absence of any force $Z_{\text {piezo }}=Z_{\text {tip }}$.

The time traces in Fig. 3 begin with $Z_{\text {piezo }}$ withdrawn $100 \mathrm{~nm}$ from the surface to a zeroed reference displacement position. As the experiment proceeds $Z_{\text {piezo }}$ is advanced towards the nanopore with $Z_{\text {tip }}$ closely following it. The ionic current through the nanopore is observed to slowly decrease as the nanotube advance initially causes a slow increase in the electrical access resistance to the pore. This trend continues until $80 \mathrm{~ms}$ into the experiment when the advancing tip displacement rate drops abruptly by a factor of $\sim 2$ relative to the $Z_{\text {piezo }}$ displacement rate. This indicates a linearly increasing mechanical resistance to the advancing $Z$ motion. There is no similar abrupt change in the ionic current at this point in time.

At $175 \mathrm{~ms}$ into the scan the ionic current starts to drop precipitously with no corresponding changes in the rates at which $Z_{\text {piezo }}$ or $Z_{\text {tip }}$ are changing. The decreasing current indicates a significantly increasing geometrical blockade to the conduction volume for electrical current transport through the nanopore. At $225 \mathrm{~ms}$ the current drop abruptly terminates and during the last $25 \mathrm{~ms}$ of advancing $Z_{\text {tip }}$ and $Z_{\text {piezo }}$ it stays remarkably constant, suggesting the nanotube has advanced all the way through the nanopore so that further advancement no longer produces a decrease in the conduction volume within the nanopore.

At $250 \mathrm{~ms}$ into the experiment the $Z_{\text {piezo }}$ motion is reversed. The subsequent ionic current and $Z_{\text {tip }}$ observations reflect a reversible (except for drift) behavior as the nanotube and cantilever are withdrawn from the nanopore. At $500 \mathrm{~ms}$ the cycle is begun again for a second threading scan. Essentially similar results are obtained in the second scan except that lateral drift causes a reduction in duration of the "total blockade" condition near $750 \mathrm{~ms}$. This trend continues in successive cycles until the nanotube drifts out of range of the pore. 
Figure 4(a) shows an expanded view of the shaded region of Fig. 3 that begins at the point where $Z_{\text {tip }}$ begins to deviate from $Z_{\text {piezo }}$. The time axis has been converted to cantilever tip displacement $Z_{\text {tip }}$ through a linear fit to curve $\mathrm{C}$ in Fig. 3 and is labeled "nanotube displacement." In the region between displacements $Z_{1}$ and $Z_{2}$ in the figure the current drops at a rate of $600 \mathrm{pA} / \mathrm{nm}$. We identify this as the range over which the nanotube tip negotiates the nanopore thickness. We find $Z_{2}-Z_{1}=9 \pm 3 \mathrm{~nm}$ which is consistent with estimates in the literature [9] based on TEM beam attenuation.

This interpretation of the data is supported by a finite element numerical simulation of the nanopore conduction current versus nanotube position shown in Fig. 4(b) (CFDACE software, ESI group, San Diego, California). We assign a conductivity $\sigma=0.1\left(\mathrm{~S} \mathrm{~cm}^{-1}\right)$ to the ionic medium and solve the steady state Poisson equation with insulating boundary conditions for a pore of diameter $D=$ $12 \mathrm{~nm}$, thickness $t=12 \mathrm{~nm}$, and a tube with $D=5 \mathrm{~nm}$. The current density throughout the solution space then follows from Ohm's law $\bar{J}=\sigma(-\nabla \phi)$. For a $200 \mathrm{mV}$ applied voltage, a base line current of $I_{o}=8.3 \mathrm{nA}$ is predicted which is within $5 \%$ of the measured value. The asymmetry between $\delta Z_{1}$ and $\delta Z_{2}$ in Fig. 4(b) (and also between $\delta I_{1}$ and $\delta I_{2}$ ) is due to the asymmetric geometry of an advancing pore entry and is consistent with the experimental data. The discrepancy between the measured and predicted total blockade current will be discussed below.

Figure 4(a) shows a high frequency oscillation impressed on the ionic current in the region defined by $Z_{2}-$ $Z_{1}$. We attribute this oscillation to an inherent lateral mechanical vibration $(\sim 500 \mathrm{~Hz}$, rms amplitude $\sim 0.5 \mathrm{~nm})$ in the $x-y$ table. These oscillations couple to the nanotube tip, conduction current, and longitudinal force on the cantilever

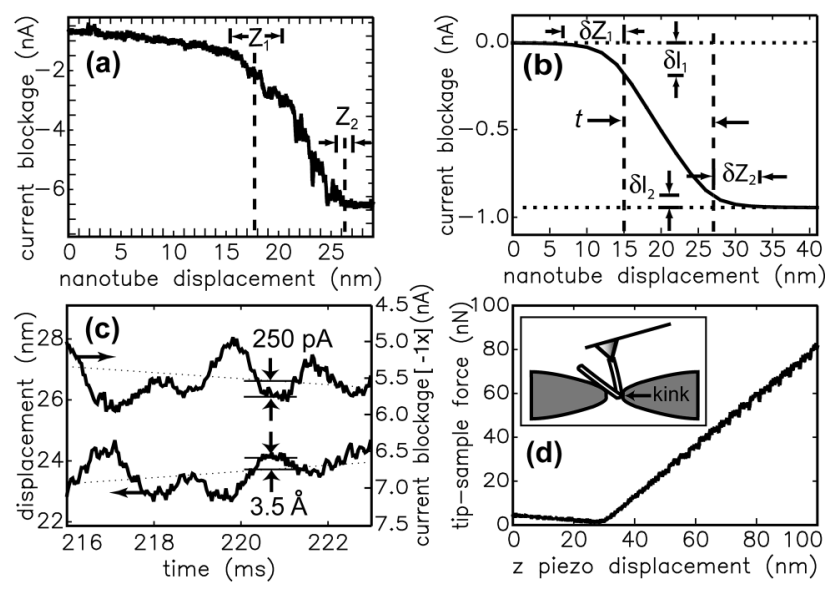

FIG. 4. (a) Ionic current blockage vs nanotube tip displacement. (b) Simulation of a nanotube translocation event. (c) Time record of ionic current and nanotube displacement. The dotted lines are local linear fits. (d) Tip-sample interaction force vs $Z_{\text {piezo }}$. Inset shows kinked nanotube translocation. due to (i) the angle ( $\sim 15$ degrees) built into the AFM cantilever, (ii) local sample slope, and (iii) any bending and/or buckling of the nanotube that couples transverse to longitudinal motion. A detailed analysis of the high frequency response of the nanopore current and the cantilever deflection shown in Fig. 4(c) demonstrates the sensitivity of the nanotube-nanopore system to extremely small changes in geometry. The figure shows that a $250 \mathrm{pA}$ change in nanopore current corresponds to 3.5 Angstrom change in nanotube tip position. Our measured rms electronic noise level is $15 \mathrm{pA}$ in a $10 \mathrm{kHz}$ bandwidth, and this leads to an effective longitudinal resolution of $0.2 \AA$ [19].

Notwithstanding the substantial evidence presented above that the nanotube threads the nanopore, there are intriguing details from the experiments for which we offer provisional explanations. For example, Fig. 4(d) shows an expanded view of the force transmitted to the cantilever during the course of the first $250 \mathrm{~ms}$ of the experiment. It is seen that $\sim 5 \mathrm{nN}$ of force is present initially and curiously this force decreases as the $z$ piezo moves the cantilever $30 \mathrm{~nm}$ towards the pore. We posit that the nanotube is actually already bent somewhere along its length when the experiment starts. This force reduction is consistent with experimentally observed nanotube kink formations in the literature [20]. In principle, kink formation occurs at the point of highest curvature of the bent tube and could be induced by the nanotube sliding along or sticking to the sample surface. Because of defects and boundary conditions at the tip and membrane surface the kink may occur anywhere along the nanotube's length. Further evidence for nanotube mechanical distortion is contained in Fig. 2. A calculation of the Euler buckling force for this nanotube [21] yields $F_{E}=34 \mathrm{nN}$. Thus, with the imaging force setpoint of $50 \mathrm{nN}$, the tube is expected to be buckled/ kinked elastically during lateral scanning $[22,23]$, which likely accounts for the $35 \mathrm{~nm}$ full width half maximum of the nanopore in Fig. 2(c) and 2(d).

At $Z_{\text {piezo }}>30 \mathrm{~nm}$, we postulate that the kinked region of the tube comes in contact with the nanopore surface, as indicated in the inset of Fig. 4(d), accounting for the abrupt change in the sign and magnitude of the slope of the force curve. From this point forward the interaction force transmitted back to the cantilever increases linearly with advancing $Z_{\text {piezo }}$. This interaction may be accounted for by mechanical nanotube bending and/or a combination of hydrophobic, van der Waal's, and short range chemical forces [24] acting between the pore's surface and the nanotube. The increasing influence of high frequency oscillations in the force versus $Z_{\text {piezo }}$ curve at $Z_{\text {piezo }}>50 \mathrm{~nm}$ can be attributable to increased coupling between the nanotube and mechanical stage oscillations as the kinked tube is forced through the pore.

The model of kinked nanotube translocation can at least partially explain the large observed maximum current blockade. Its measured value is roughly 7 times larger 
than expected for the pore and nanotube cross sectional areas. A threaded kinked tube blocks a larger area than an unkinked one by at least a factor of 2 , and likely more due to components of the kinked nanotube surface transverse to the longitudinal axis of the nanopore [25]. Further potential explanations for the large observed blockage current include the existence of a nonconducting surface layer of water around the nanotube [26], charge (e.g., bound ions) residing on the nanotube, and/or a contamination layer accumulated on the tube acquired when it passes through the liquid-air interface.

We conclude with a few remarks regarding the potential utility of the results presented above. Recently Zheng et al. [27] demonstrated that carbon nanotubes naturally bind single stranded DNA molecules. By deterministically translocating these molecules, affixed to nanotubes, through nanopore devices it may well be possible to probe and perhaps sequence such molecules. As opposed to electrophoretic nanopore translocation methods, here the molecules need not be charged which enhances the range of molecules that can be studied. A new approach to single molecule "molecular electronics" studies requires nanopores that are articulated with stationary electrical probes at their periphery. This structure, with an electrically contacted threading nanotube articulated with molecules, will allow local electronic properties to be probed by tunneling during translocation in the nanopore. We thus envision that nanotube threaded nanopore devices will enable many interesting measurement opportunities for the study of the electrical and ionic transport properties of molecules.

We acknowledge support from DARPA, NSF, DOE, and Agilent Technologies. We wish to thank Professor Dan Branton, Dr. Gregor Schürmann, Eric Brandin, James Dunning, and Haibing Peng for their valuable assistance and advice.

[1] G. Binnig et al., Phys. Rev. Lett. 50, 120 (1983).

[2] G. Binnig, C. Quate, and C. Gerber, Phys. Rev. Lett. 56, 930 (1986).

[3] P. K. Hansma et al., Science 243, 641 (1989).
[4] R. Wiesendanger, Scanning Probe Microscopy and Spectroscopy (Cambridge University Press, Cambridge, England, 1994).

[5] M. Dresselhaus, G. Dresselhaus, and P. Eklund, Science of Fullerenes and Carbon Nanotubes (Academic, New York, 1995).

[6] B. Hille, Ionic Channels and Excitable Membranes (Sinauer, Sunderland, Massachusetts, 1992).

[7] J. J. Kasianowicz et al., Proc. Natl. Acad. Sci. U.S.A. 93, 13770 (1996).

[8] S. Howorka et al., Proc. Natl. Acad. Sci. U.S.A. 98, 12996 (2001).

[9] J. Li et al., Nature (London) 412, 166 (2001).

[10] J. Li et al., Nat. Mater. 2, 611 (2003).

[11] S.S. Narayan et al., Science 282, 1882 (1998).

[12] E. Yenilmez et al., Appl. Phys. Lett. 80, 2225 (2002).

[13] CSC21 triangular cantilevers, MikroMasch USA, Portland, Oregon.

[14] H. Peng et al., Appl. Phys. Lett. 83, 4238 (2003).

[15] M. Yu et al., Science 287, 637 (2000).

[16] The $\sim 5 \mathrm{~nm}$ topographic rise around the nanopore region is a ubiquitous feature of the ion sculpting method and will be discussed elsewhere.

[17] This value is only approximate because absolute position determination in AFM between a disengaged tip and sample is unreliable. Our average $z$ drift rate in ionic solution is $\sim 0.35 \mathrm{~nm} / \mathrm{s}$ in $-z$ (i.e., the tip drifts towards the surface).

[18] The measured lateral drift rate of our AFM under ionic fluid is $\sim 0.5 \mathrm{~nm} / \mathrm{s}$. In practice the necessary nanoscale alignment allowing full nanotube translocation persists for $\sim 2$ s.

[19] Analogous measurements of a larger pore $(D=30 \mathrm{~nm})$ with a different nanotube tip $(D=3 \mathrm{~nm})$ generated a longitudinal resolution of $0.8 \AA$. Clearly smaller pores yield higher resolution.

[20] E. Wong, P. Sheehan, and C. Lieber, Science 277, 1971 (1997).

[21] H. Dai et al., Nature (London) 384, 147 (1996).

[22] H. Dai, N. Franklin, and J. Han, Appl. Phys. Lett. 73, 1508 (1998).

[23] Our measured force versus distance data is reproducible and reversible, and thus consistent with elastic buckling.

[24] T. Hertel, R. Martel, and P. Avouris, J. Phys. Chem. B 102, 910 (1998).

[25] M. R. Falvo et al., Nature (London) 389, 582 (1997).

[26] J. Walther et al., Carbon 42, 1185 (2004).

[27] M. Zheng et al., Science 302, 1545 (2003). 